\title{
Global Solvability of a Continuous Model for Nonlocal Fragmentation Dynamics in a Moving Medium
}

\author{
S. C. Oukouomi Noutchie and E. F. Doungmo Goufo \\ Department of Mathematical Sciences, North-West University, Mafikeng 2735, South Africa \\ Correspondence should be addressed to E. F. Doungmo Goufo; franckemile2006@yahoo.ca
}

Received 27 March 2013; Accepted 23 May 2013

Academic Editor: Guo-Cheng Wu

Copyright (c) 2013 S. C. Oukouomi Noutchie and E. F. Doungmo Goufo. This is an open access article distributed under the Creative Commons Attribution License, which permits unrestricted use, distribution, and reproduction in any medium, provided the original work is properly cited.

\begin{abstract}
Existence of global solutions to continuous nonlocal convection-fragmentation equations is investigated in spaces of distributions with finite higher moments. Under the assumption that the velocity field is divergence-free, we make use of the method of characteristics and Friedrichs's lemma (Mizohata, 1973) to show that the transport operator generates a stochastic dynamical system. This allows for the use of substochastic methods and Kato-Voigt perturbation theorem (Banasiak and Arlotti, 2006) to ensure that the combined transport-fragmentation operator is the infinitesimal generator of a strongly continuous semigroup. In particular, we show that the solution represented by this semigroup is conservative.
\end{abstract}

\section{Motivation and Introduction}

The process of fragmentation of clusters occurs in many branches of natural sciences ranging from physics, through chemistry, engineering, biology, to ecology and in numerous domains of applied sciences, such as the depolymerization, the rock fractures, and the breakage of droplets. The fragmentation rate can be size and position dependent, and new particles resulting from the fragmentation are spatially distributed across the space. Fragmentation equations, combined with transport terms, have been used to describe a wide range of phenomena. For instance, in ecology or aquaculture, we have phytoplankton population in flowing water. In chemical engineering, we have applications describing polymerization, polymer degradation, and solid drugs breakup in organisms or in solutions. We also have external processes such as oxidation, melting, or dissolution, which cause the exposed surface of particles to recede, resulting in the loss of mass of the system. Simultaneously, they widen the surface pores of the particle, causing the loss of connectivity and thus fragmentation, as the pores join each other (see [1-4] and references therein). Various types of pure fragmentation equations have been comprehensively analyzed in numerous works (see, e.g., [5-9]). Conservative and nonconservative regimes for pure fragmentation equations have been thoroughly investigated, and, in particular, the breach of the mass conservation law (called shattering) has been attributed to a phase transition creating a dust of "zero-size" particles with nonzero mass, which are beyond the model's resolution. But fragmentation and transport processes combined in the same model are still barely touched in the domain of mathematical and abstract analysis. Kinetic-type models with diffusion were globally investigated in [5] and later extended in [10], where the author showed that the diffusive part does not affect the breach of the conservation laws, and, very recently, in [11], the author investigated equations describing fragmentation and coagulation processes with growth or decay and proved an analogous result.

In this paper, we present and analyze a special and noncommon type of transport process. In social grouping population, if we define a spatial dynamical system in which locally group-size distribution can be estimated, but in which we also allow immigration and emigration from adjacent areas with different distributions, we obtain the general model consisting of transport, direction changing, and fragmentation and coagulation processes describing the dynamics a population of, for example, phytoplankton, which is a spatially explicit group-size distribution model 
as presented in [12]. We analyze, in this work, the model consisting of transport and fragmentation processes, hoping that it will bring a significant contribution to the analysis of the full problem (with transport, direction changing, and fragmentation and coagulation processes) which remains an open problem.

\section{Well Posedness of the Transport Problem with Fragmentation}

We consider the following Cauchy problem [12]:

$$
\begin{aligned}
& \frac{\partial}{\partial t} p(t, x, m) \\
&=-\operatorname{div}(\omega(x, m) p(t, x, m)) \\
&-a(x, m) p(t, x, m) \\
&+\int_{m}^{\infty} b(x, s, m) a(x, s) p(t, x, s) d s, \\
& p(0, x, m)=\stackrel{o}{p}(x, m), \quad \text { a.e. }(x, m) \in \mathbb{R}^{3} \times \mathbb{R}_{+},
\end{aligned}
$$

where, in terms of the mass size $m$ and the position $x$, the state of the system is characterized at any moment $t$ by the particle-mass-position distribution $p=p(t, x, m)$ ( $p$ is also called the density or concentration of particles), with $p: \mathbb{R}_{+} \times$ $\mathbb{R}^{3} \times \mathbb{R}_{+} \rightarrow \mathbb{R}_{+}$. The three-dimensional vector $\omega=\omega(x, m)$ represents the velocity of the transport and is supposed to be a known quantity depending on $m$ and $x ; a(x, m)$ is the average fragmentation rate; that is, it describes the ability of aggregates of size $m$ and position $x$ to break into smaller particles. Once an aggregate of mass $s$ and position $x$ breaks, the expected number of daughter particles of size $m$ is the nonnegative measurable function $b(x, s, m)$ defined on $\mathbb{R}^{3} \times$ $\mathbb{R} \times \mathbb{R}$. The space variable $x$ is supposed to vary in the whole of $\mathbb{R}^{3}$. The function $\stackrel{o}{p}(x, m)$ represents the density of groups of size $m$ at position $x$ at the beginning $(t=0)$.

2.1. Fragmentation Equation. Let us introduce necessary assumptions that will be useful in our analysis. Since a group of size $m \leq s$ cannot split to form a group of size $s$, the function $b(x, s, m)$ has its support in the set

$$
\mathbb{R}^{3} \times\left\{(s, m) \in \mathbb{R}_{+} \times \mathbb{R}_{+}: m<s\right\} .
$$

After the fragmentation of a mass $s$ particle, the sum of masses of all daughter particles should again be $s$; hence it follows that, for any $s>0, x \in \mathbb{R}^{3}$

$$
\int_{0}^{s} m b(x, s, m) d m=s .
$$

Because the space variable $x$ varies in the whole of $\mathbb{R}^{3}$ (unbounded) and since the total number of individuals in a population is not modified by interactions among groups, the following conservation law is supposed to be satisfied:

$$
\frac{d}{d t} \mathcal{N}(t)=0
$$

where $\mathcal{N}(t)=\int_{\mathbb{R}^{3}} \int_{0}^{\infty} p(t, x, m) m d m d x$ is the total number of individuals in the space (or total mass of the ensemble). Since $p=p(t, x, m)$ is the density of groups of size $m$ at the position $x$ and time $t$ and that mass is expected to be a conserved quantity, the most appropriate Banach space to work in is the space

$$
\mathscr{X}_{1}:=L_{1}\left(\mathbb{R}^{3} \times \mathbb{R}_{+}, m d m d x\right)
$$

But because uniqueness of solutions of (1) proved to be a more difficult problem [11], we restrict our analysis to a smaller class of functions, so we introduce the following class of Banach spaces (of distributions with finite higher moments):

$$
\mathscr{X}_{r}:=L_{1}\left(\mathbb{R}^{3} \times \mathbb{R}_{+}, m^{r} d m d x\right), \quad r \geq 1,
$$

which coincides with $\mathscr{X}_{1}$ for $r=1$ and is endowed with the norm $\|\cdot\|_{r}$. We assume that $\stackrel{o}{p} \in \mathscr{X}_{r}$, and, for each $t \geq 0$, the function $(x, m) \rightarrow p(x, m)=p(t, x, m)$ is from the space $\mathscr{X}_{r}$ with $r \geq 1$. When any subspace $S \subseteq \mathscr{X}_{r}$, we will denote by $S_{+}$the subset of $S$ defined as $S_{+}=\{g \in S ; g(x, m) \geq 0, m \in$ $\left.\mathbb{R}_{+}, x \in \mathbb{R}^{3}\right\}$. Note that any $g \in\left(\mathscr{X}_{r}\right)_{+}$will possess moments

$$
M_{q}(t):=\int_{0}^{\infty} m^{q} g(t, x, m) d m
$$

of all orders $q \in[0, r]$. In $\mathscr{X}_{r}$, we define from the expressions on the right-hand side of (1) the operators $A$ and $B$ by

$$
\begin{gathered}
{[A g](x, m):=a(x, m) g(x, m),} \\
D(A):=\left\{g \in \mathscr{X}_{r}: a g \in \mathscr{X}_{r}\right\}, \\
{[B g](x, m):=\int_{m}^{\infty} b(x, s, m) a(x, s) p(x, s) d s,} \\
D(B):=D(A) .
\end{gathered}
$$

Lemma 1. $(A+B, D(A))$ is a well-defined operator.

Proof. To prove that $B$ is well defined on $D(A)$ as stated in (9), we use the condition (3) to show that

$$
\begin{aligned}
s^{r}-\int_{0}^{s} m^{r} b(x, s, m) d m & =s^{r}-\int_{0}^{s} m^{r-1} m b(x, s, m) d m \\
& \geq s^{r}-s^{r-1} \int_{0}^{s} m b(x, s, m) d m=0 .
\end{aligned}
$$

Hence

$$
\int_{0}^{s} m^{r} b(x, s, m) d m \leq s^{r}
$$


for $r \geq 1, m>0$. Note that the equality holds for $r=1$. For every $p \in D(A)_{+}$, changing the order of integration by the Fubini theorem, we have

$$
\begin{aligned}
& \|B p\|_{r} \\
& \quad=\int_{\mathbb{R}^{3}} \int_{0}^{\infty}[B p](x, m) m^{r} d m d x \\
& =\int_{\mathbb{R}^{3}} \int_{0}^{\infty}\left(\int_{m}^{\infty} b(x, s, m) a(x, s) p(x, s) m^{r} d s\right) d m d x \\
& =\int_{\mathbb{R}^{3}} \int_{0}^{\infty}\left(\int_{0}^{s} b(x, s, m) a(x, s) p(x, s) m^{r} d m\right) d s d x \\
& \quad \leq \int_{\mathbb{R}^{3}} \int_{0}^{\infty} a(x, s) p(x, s) s^{r} d s d x \\
& =\|A p\|_{r} \\
& \quad<\infty
\end{aligned}
$$

where we have used inequality (11). The result follows from the fact that any arbitrary element $p$ of $D(A)$ can be written in the form $p=p_{+}-p_{-}$, where $p_{+}, p_{-} \in D(A)_{+}$. Then $\|B p\|_{r} \leq$ $\|A p\|_{r}$, for all $p \in D(A)$, so that we can take $D(B):=D(A)$, and $(A+B, D(A))$ is well defined.

2.2. Cauchy Problem for the Transport Operator in $\Lambda=\mathbb{R}^{3} \times$ $\mathbb{R}_{+} \cdot \Lambda$ is endowed with the Lebesgue measure $d \mu=d \mu_{m, x}=$ $d m d x$. Our primary objective in this section is to analyze the solvability of the transport problem

$$
\begin{gathered}
\frac{\partial}{\partial t} p(t, x, m)=-\operatorname{div}(\omega(x, m) p(t, x, m)), \\
p(0, x, m)=\stackrel{o}{p}(x, m), \quad m \in \mathbb{R}_{+}, x \in \mathbb{R}^{3}
\end{gathered}
$$

in the space $\mathscr{X}_{r}$.

Furthermore, to simplify the notation we put $\mathbf{v}=(x, m) \epsilon$ $\Lambda$. We consider the function $\omega: \Lambda \rightarrow \mathbb{R}^{3}$ and $\widetilde{\mathscr{D}}$ the expression appearing on the right-hand side of (13). Then

$$
\begin{aligned}
\widetilde{D}[p(t, \mathbf{v})]: & =-\operatorname{div}(\omega(\mathbf{v}) p(t, \mathbf{v})) \\
& =(\nabla \cdot \omega(\mathbf{v})) p(t, \mathbf{v})+\omega(\mathbf{v}) \cdot(\nabla p(t, \mathbf{v})) .
\end{aligned}
$$

We assume that $\omega$ is divergence-free and globally Lipschitz continuous. Then $\operatorname{div} \omega(\mathbf{v}):=\nabla \cdot \omega(\mathbf{v})=0$, and (14) becomes

$$
\widetilde{\mathscr{D}}[p(t, \mathbf{v})]:=\omega(\mathbf{v}) \cdot(\nabla p(t, \mathbf{v})) .
$$

For $\mathbf{v} \in \Lambda$ and $t \in \mathbb{R}$, the initial value problem

$$
\begin{gathered}
\frac{d \mathfrak{r}}{d s}=\omega(\mathfrak{r}), \quad s \in \mathbb{R}, \\
\mathfrak{r}(t)=\mathbf{v}
\end{gathered}
$$

has one and only one solution $\mathfrak{r}(s)$ taking values in $\Lambda$. Thus we can consider the function $\phi: \Lambda \times \mathbb{R}^{2} \rightarrow \Lambda$ defined by the condition that, for $(\mathbf{v}, t) \in \Lambda \times \mathbb{R}$,

$$
s \longrightarrow \phi(\mathbf{v}, t, s), \quad s \in \mathbb{R},
$$

is the only solution of the Cauchy problem (16). The integral curves given by the $\phi$-parameter family $(\mathfrak{r})_{\phi}$ (with $\mathfrak{r}(s)=$ $\phi(\mathbf{v}, t, s), s \in \mathbb{R}$, the only solution of (16)) are called the characteristics of $\widetilde{\mathscr{D}}$. The function $\phi$ possesses many desirable properties [13-15] that will be relevant for studying the transport operator in $\mathscr{X}_{r}$. Some of them are listed in $[5$, Proposition 10.1]. Now we can properly study the transport operator $\mathscr{D}$. Using the above proposition in our application, we can take

$$
\begin{gathered}
\mathscr{D} p=\widetilde{\mathscr{D}}_{p}, \quad \text { with } \widetilde{\mathscr{D}}_{p} \text { represented by (15), } \\
D(\mathscr{D}):=\left\{p \in \mathscr{X}_{r}, \mathscr{D} p \in \mathscr{X}_{r}\right\} .
\end{gathered}
$$

Note that $\mathscr{D} p$ is understood in the sense of distribution. Precisely speaking, if we take $C_{0}^{1}(\Lambda)$ as the set of the test functions, each $p \in D(\mathscr{D})$ if and only if $p \in L_{1}(\Lambda)$, and there exists $g \in \mathscr{X}_{r}$ such that

$$
\int_{\Lambda} \xi g d \mu=\int_{\Lambda} p \partial \cdot(\xi \omega) d \mu=\int_{\Lambda} p \omega \cdot \partial \xi d \mu,
$$

for all $\xi \in C_{0}^{1}(\Lambda)$, where

$$
\omega \cdot \partial \xi(\mathbf{v}):=\sum_{j=1}^{3} \omega_{j} \partial_{j} \xi(\mathbf{v})
$$

with $\omega_{j}=\omega_{j}(\mathbf{v})$, the $j$ th component of the velocity $\omega(\mathbf{v})$. The middle term in (19) exists as $\omega$ is globally Lipschitz continuous, and the last equality follows as $\omega$ is divergencefree. If this is the case, we define $\mathscr{D} p=g$.

Now we can show that the operator $\mathscr{D}$ is the generator of a stochastic semigroup on $\mathscr{X}_{r}$.

Theorem 2. If the function $\omega$ is globally Lipschitz continuous and divergence-free, then the operator $(D(\mathscr{D}), \mathscr{D})$ defined by (18) is the generator of a strongly continuous stochastic semigroup $\left(G_{\mathscr{D}}(t)\right)_{t \geq 0}$, given by

$$
\left[G_{\mathscr{D}}(t) p\right](\mathbf{v})=p(\phi(\mathbf{v}, t, 0))
$$

for any $p \in \mathscr{X}_{r}$ and $t>0$.

Proof. Let $\left(Z_{0}(t)\right)_{t \geq 0}$ be the family defined by the right-hand side of the relation (21). The proof of the theorem will follow three steps.

(i) First we show that $\left(Z_{0}(t)\right)_{t \geq 0}$ is a strongly continuous semigroup of bounded linear operators. We need some properties of $\phi$ as listed in [5] and given as follows. The function $\phi$ has the following properties:

$\left(p_{1}\right) \phi(\mathbf{v}, t, t)=\mathbf{v}$ for all $\mathbf{v} \in \Lambda, t \in \mathbb{R} ;$

$\left(p_{2}\right) \phi(\phi(\mathbf{v}, t, s), s, \tau)=\phi(\mathbf{v}, t, \tau)$ for all $\mathbf{v} \in \Lambda, t, s$, and $\tau \in$ $\mathbb{R}$; 
$\left(p_{3}\right) \phi(\mathbf{v}, t, s)=\phi(\mathbf{v}, t-s, 0)=\phi(\mathbf{v}, 0, s-t)$ for all $\mathbf{v} \in \Lambda$, $t, s \in \mathbb{R}$

$\left(p_{4}\right)|\phi(\mathbf{v}, t, s)-\phi(\mathbf{y}, t, s)| \leq e^{K|t-s|}|\mathbf{v}-\mathbf{y}|$ for all $\mathbf{v}, \mathbf{y} \in \Lambda$, $t, s \in \mathbb{R}$

$\left(p_{5}\right)$ function $\Lambda \times \mathbb{R} \times \mathbb{R} \ni(\mathbf{v}, t, s) \rightarrow \phi(\mathbf{v}, t, s)$ is continuous;

$\left(p_{6}\right)$ the transformation $\mathscr{T}$ defined by $t=t, s=s$, and $\mathbf{y}=\phi(\mathbf{v}, t, s)$ is a topological homeomorphism which is bimeasurable, and its inverse $\mathscr{T}^{-1}$ is represented by $t=t, s=s$, and $\mathbf{v}=\phi(\mathbf{y}, s, t) ;$

$\left(p_{7}\right)$ for all $t, s \in \mathbb{R}$ the transformation of $\Lambda$ onto itself defined by $\mathbf{y}=\phi(\mathbf{v}, t, s)$ is measure preserving.

Then by the properties $\left(p_{6}\right)$ and $\left(p_{7}\right)$, we see that, for any $p$, the composition $(\mathbf{v}, t) \rightarrow p(\phi(\mathbf{v}, t, 0))$, in (21), is a measurable function satisfying the equality

$$
\left\|Z_{0}(t) p\right\|_{r}=\|p\|_{r} .
$$

Hence the family $\left(Z_{0}(t)\right)_{t \geq 0}$ is of bounded linear operators from $\mathscr{X}_{r} \rightarrow \mathscr{X}_{r}$. Then we can easily verify the following relations:

$$
\begin{aligned}
& \left(i_{a}\right) Z_{0}(0)=I ; \\
& \left(i_{b}\right) Z_{0}(t+s)=Z_{0}(t) Z_{0}(s) \text {, for all } t, s \in \mathbb{R} ; \\
& \left(i_{c}\right) \lim _{t \rightarrow 0^{+}}\left\|Z_{0}(t) p-p\right\|_{r}=0 \text {, for each } p \in X_{r} .
\end{aligned}
$$

In fact, $\left(i_{a}\right)$ and $\left(i_{b}\right)$ follow immediately from the properties $\left(p_{1}\right)$ and $\left(p_{2}\right)$. To prove $\left(i_{c}\right)$, we can follow the argument of Example 3.10 in [5]. Thus, it is enough to show $\left(i_{c}\right)$ for $p \in$ $C_{0}^{\infty}(\Lambda)$. For such $p_{s}$, we have $\lim _{t \rightarrow 0^{+}}\left(Z_{0}(t) p\right)(\mathbf{v})=p(\mathbf{v})$ for all $\mathbf{v} \in \Lambda$. Furthermore, if $|p(\mathbf{v})| \leq M$ for all $\mathbf{v} \in \Lambda$, then $\left|\left(Z_{0}(t) p\right)(\mathbf{v})\right| \leq M$ for all $\mathbf{v} \in \Lambda$, and, because the support of $Z_{0}(t) p$ is bounded, the Lebesgue dominated convergence theorem shows that $\left(i_{c}\right)$ is satisfied. Thus $\left(Z_{0}(t)\right)_{t \geq 0}$ is a $C_{0^{-}}$ semigroup.

(ii) Secondly, we prove that the generator $T_{0}$ of $\left(Z_{0}(t)\right)_{t \geq 0}$ is an extension of $\mathscr{D}$.

Let $\mathcal{Y}$ be the set of real-valued functions which are defined on $\Lambda$, are Lipschitz continuous, and compactly supported. Obviously $\mathcal{Y} \subset D(\mathscr{D})$ because if $p \in \mathcal{Y}$, then the first-order partial derivatives of $p$ are measurable, bounded, and compactly supported and thus, multiplied by Lipschitz continuous functions of $\omega$, belong to $L_{1}(\Lambda, d \mu)$. For any fixed $p \in \mathcal{Y}$, we denote by $\vartheta$ the real-valued function defined on $\Lambda \times R_{+}$by

$$
\vartheta(\mathbf{v}, t)=\left(Z_{0}(t) p\right)(\mathbf{v}) .
$$

From the previous considerations and properties $\left(p_{3}\right)-\left(p_{5}\right)$ there exists a measurable subset $E$ of $\Lambda \times R_{+}$, with $\mu\left(\Lambda \times R_{+} \backslash\right.$ $E)=0$, such that at each point $(\mathbf{v}, t) \in E$ the function $\vartheta$ has measurable first-order partial derivatives. In particular,

$$
\frac{\partial \vartheta}{\partial t}(\mathbf{v}, t)=\left(Z_{0}(t) \mathscr{D} p\right)(\mathbf{v}), \quad(\mathbf{v}, t) \in E,
$$

and, therefore, if we let $\lambda_{p}:=\operatorname{ess} \sup _{(\mathrm{v}) \in \Lambda}|\mathscr{D} p|$, then

$$
\left|\partial_{t} \vartheta(\mathbf{v}, t)\right| \leq \lambda_{p}
$$

for any $(\mathbf{v}, t) \in E$. From this and from part (i) of the proof it follows that, for every $h>0$,

$$
\begin{aligned}
& \left\|h^{-1}\left(Z_{0}(h) p-p\right)-\mathscr{D} p\right\|_{r} \\
& \quad=\left\|h^{-1} \int_{0}^{h}\left(Z_{0}(s)-I\right) \mathscr{D} p d s\right\|_{r} \longrightarrow 0
\end{aligned}
$$

as $h \rightarrow 0^{+}$. This proves that $\mathscr{Y} \subset D\left(T_{0}\right)$ and that $T_{0} p=\mathscr{D} p$, for all $p \in \mathscr{Y}$. Next we prove that $\mathscr{Y}$ is a core of $\mathscr{D}$, that is, that $(\mathscr{D}, D(\mathscr{D}))$ is the closure of $(\mathscr{D}, \mathscr{Y})$. Let $\omega_{\varepsilon}, \varepsilon>0$, be a mollifier (see Example 2.1 in [5]), and, for $p$, let $\omega_{\varepsilon} * p$ be the mollification of $p$. We use the Friedrichs lemma, [16, pp. 313315], or [17, Lemma 1.2.5], which states that there is $C>0$, independent of $\varepsilon$, such that for any $L_{r}$ function $p, 1 \leq r<\infty$, we have

$$
\begin{gathered}
\left\|\mathscr{D}\left(\omega_{\varepsilon} * p\right)-\omega_{\varepsilon} * \mathscr{D} p\right\|_{r} \leq C\|p\|_{r}, \\
\lim _{\varepsilon \rightarrow 0^{+}}\left(\left\|\omega_{\varepsilon} * p-p\right\|_{r}+\left\|\mathscr{D}\left(\varrho_{\varepsilon} * p\right)-\mathscr{D} p\right\|_{r}\right)=0 .
\end{gathered}
$$

Estimates of Equation (2.9) in [5] and the above relation (27) imply

$$
\left\|\mathscr{D}\left(\varrho_{\varepsilon} * p\right)\right\|_{r} \leq C\|p\|_{r}+\|\mathscr{D} p\|_{r}
$$

which shows that the mollification $p \rightarrow \omega_{\varepsilon} * p$ is a continuous operator in $D(\mathscr{D})$ (equipped with the graph norm) uniformly bounded with respect to $\varepsilon$. Next we observe that the subset of $D(\mathscr{D})$ consisting of compactly supported functions is dense in $D(\mathscr{D})$ with the graph norm. Indeed, let $p \in D(\mathscr{D})$. Because both $p, \mathscr{D} p \in \mathscr{X}_{r}$, the absolute continuity of the Lebesgue integral implies that for any given $\delta>0$ there exists a compact subset $\Omega^{\prime}$ of $\Lambda$ such that

$$
\int_{\Lambda \mid \Omega^{\prime}}(|p|+|\mathscr{D} p|) d \mu<\delta
$$

For this $\Omega^{\prime}$ we choose $\psi \in C_{0}^{\infty}(\Lambda)$ satisfying $0 \leq \psi(\mathbf{v}) \leq 1$ for all $\mathbf{v} \in \Lambda$, and $\psi(\mathbf{v})=1$ for all $\mathbf{v} \in \Omega^{\prime}$. Now it is easy to see that $\psi p \in D(\mathscr{D})$ and has a compact support. Moreover,

$$
\begin{gathered}
\|\psi p-p\|_{r} \leq 2 \int_{\Lambda \mid \Omega^{\prime}}|p| d \mu, \\
\|\mathscr{D}(\psi p)-\mathscr{D} p\|_{r} \leq 2 \int_{\Lambda \mid \Omega^{\prime}}|\mathscr{D} p| d \mu+L \int_{\Lambda \mid \Omega^{\prime}}|p| d \mu,
\end{gathered}
$$

where $L=\sup |D \psi|$ can be made independent of $\Omega^{\prime}$ due to the fact that $\Lambda$ is the whole space.

Let $p \in D(\mathscr{D})$ be compactly supported. From Example 2.1 in [5] we know that $\omega_{\varepsilon} * p$ is infinitely differentiable and compactly supported and thus belongs to $\mathcal{Y}$. Equation (28) yields that $\omega_{\varepsilon} * p \rightarrow p$ as $\varepsilon \rightarrow 0^{+}$in the graph norm of $D(\mathscr{D})$. Because we have shown above that compactly supported functions from $D(\mathscr{D})$ are dense in $D(\mathscr{D})$, we see that $(\mathscr{D}, D(\mathscr{D}))$ is the closure of $(\mathscr{D}, \mathscr{Y})$, and, because $T_{0}$ is a closed extension of $(\mathscr{D}, \mathscr{Y})$, we obtain $\mathscr{D} \subset T_{0}$.

(iii) Lastly we recognize that $D\left(T_{0}\right) \subset D(\mathscr{D})$ so that the operators $T_{0}$ and $\mathscr{D}$ coincide, and $\left(G_{\mathscr{D}}(t)\right)_{t \geq 0}=\left(Z_{0}(t)\right)_{t \geq 0}$. 
Suppose $p \in D\left(T_{0}\right)$. Then for any fixed $\lambda>0$ there exists a unique $g \in \mathscr{X}_{r}$ such that $p=\left(\lambda I-T_{0}\right)^{-1} g$. For any $\psi \in C_{0}^{1}(\Lambda)$ we have, by (19),

$$
\begin{aligned}
\int_{\Lambda} & \mathscr{D} p \psi d \mu \\
= & \int_{\Lambda} p(\mathbf{v})(\omega \cdot \partial \psi)(\mathbf{v}) d \mu_{\mathbf{v}} \\
= & \int_{\Lambda}\left(\int_{0}^{\infty} e^{-\lambda t} g(\phi(\mathbf{v}, t, 0)) d t\right)(\omega \cdot \partial \psi)(\mathbf{v}) d \mu_{\mathbf{v}} \\
= & \int_{0}^{\infty}\left(\int_{\Lambda} e^{-\lambda t} g(\phi(\mathbf{v}, t, 0))(\omega \cdot \partial \psi)(\mathbf{v}) d \mu_{\mathbf{v}}\right) d t \\
= & \int_{0}^{\infty}\left(\int_{\Lambda} e^{-\lambda t} g(\mathbf{y})(\omega \cdot \partial \psi)(\phi(\mathbf{y}, 0, t))(\mathbf{v}) d \mu_{\mathbf{y}}\right) d t \\
= & \int_{\Lambda}\left(\int_{0}^{\infty} e^{-\lambda t} \frac{d}{d t} \psi(\phi(\mathbf{y}, 0, t)) d t\right) g(\mathbf{y}) d \mu_{\mathbf{y}} \\
= & \int_{\Lambda}\left(\left.e^{-\lambda t} \psi(\phi(\mathbf{y}, 0, t))\right|_{0} ^{\infty} g(\mathbf{y}) d y\right. \\
& \left.+\lambda \int_{\Lambda}\left(\int_{0}^{\infty} e^{-\lambda t} \psi(\phi(\mathbf{y}, 0, t)) d t\right)\right) g(\mathbf{y}) d \mu_{\mathbf{y}} \\
= & -\int_{\Lambda}(g-\lambda p) \psi d \mu . \\
& \left.+\lambda \int_{\Lambda}\left(\int_{0}^{\infty} e^{-\lambda t} g\left(\phi(\mathbf{y}) \psi(\mathbf{y}) d \mu_{\mathbf{y}}, t\right)\right) d t\right) \psi(\mathbf{v}) d \mu_{\mathbf{v}}
\end{aligned}
$$
$T_{0} p$.

This implies that $p \in D(\mathscr{D})$. Hence $T_{0} \subset \mathscr{D}$, and $\mathscr{D} p=$

Remark 3 (conservativeness of the transport model). Because the flow process does not modify the total number of individuals in the system, let us show that the model (13) is conservative in the space $\mathscr{X}_{r}$; that is, the law (4) is satisfied. We have proved that the semigroup generated by the operator $\mathscr{D}$ is stochastic; then we have

$$
\begin{gathered}
0=\int_{\Lambda} \mathscr{D} p d \mu, \quad \forall p \in D(\mathscr{D}), \text { then } \\
0=\int_{\mathbb{R}^{3}} \int_{0}^{\infty} m^{r} \mathscr{D} p(t, x, m) d m d x, \quad \forall t \geq 0, r \geq 1 .
\end{gathered}
$$

Thus, $\int_{\mathbb{R}^{3}} \int_{0}^{\infty} m \mathscr{D} p(t, x, m) d m d x=0$, for all $t \geq 0$ which leads to

$$
\begin{aligned}
\frac{d}{d t} \mathcal{N}(t) & =\frac{d}{d t}\left(\int_{\mathbb{R}^{3}} \int_{0}^{\infty} m p(t, x, m) d m d x\right) \\
& =\int_{\mathbb{R}^{3}} \int_{0}^{\infty} m \partial_{t} p(t, x, m) d m d x
\end{aligned}
$$

$$
\begin{aligned}
& =\int_{\mathbb{R}^{3}} \int_{0}^{\infty} m \mathscr{D} p(t, x, m) d m d x \\
& =0
\end{aligned}
$$

and therefore proving the conservativeness of the transport model in (18).

\section{Perturbed Transport-Fragmentation Problems}

We turn now to the transport problem with the loss part of the fragmentation process. We assume that there are two constants $0<\theta_{1}$ and $\theta_{2}$ such that for every $x \in \mathbb{R}^{3}$,

$$
\theta_{1} \alpha_{m} \leq a(x, m) \leq \theta_{2} \alpha_{m}
$$

with $\alpha_{m} \in \mathbb{R}_{+}$and independent of $x$. Then we can consider the loss operator $(A, D(A))$ defined in (8). The corresponding abstract Cauchy problem reads as

$$
\begin{aligned}
\partial_{t} p(t, \mathbf{v}) & =\mathscr{D} p(t, \mathbf{v})-A p(t, \mathbf{v})=F p(t, \mathbf{v}), \\
p(0, \mathbf{v}) & =\stackrel{o}{p}(\mathbf{v}), \quad \mathbf{v} \in \Lambda,
\end{aligned}
$$

where

$$
F=\mathscr{D}-A .
$$

We provide a characterization of the domain $D(F)$.

Lemma 4. Consider $D(F)=D(\mathscr{D}) \cap D(A)(=D(\mathscr{D}))$.

Proof. First of all it is obvious to see that $D(\mathscr{D}) \cap$ $D(A)=D(\mathscr{D})$ since $D(A)=\mathscr{X}_{r}$. Because $\mathscr{D}$ is con servative, integration of (36) over $\Lambda$ gives $(d / d t)\|p\|_{r}=$ $(d / d t) \int_{\mathbb{R}^{3}} \int_{0}^{\infty} m^{r} p(t, x, m) d m d x=-\int_{\mathbb{R}^{3}} \int_{0}^{\infty} a(x, m) m^{r} p(x$, $m) d m d x$. Hence (35) leads to

$$
\begin{aligned}
-\int_{\mathbb{R}^{3}} & \int_{0}^{\infty} \theta_{2} \alpha_{m} m^{r} p(x, m) d m d x \\
& \leq-\int_{\mathbb{R}^{3}} \int_{0}^{\infty} a(x, m) m^{r} p(x, m) d m d x \\
& \leq-\int_{\mathbb{R}^{3}} \int_{0}^{\infty} \theta_{1} \alpha_{m} m^{r} p(x, m) d m d x
\end{aligned}
$$

for all $p \in\left(\mathscr{X}_{r}\right)_{+}$, and, using Gronwall's inequality, we obtain

$$
-\theta_{2} \alpha_{m}\|p\|_{r} \leq \frac{d}{d t}\|p\|_{r} \leq-\theta_{1} \alpha_{m}\|p\|_{r} .
$$

Then

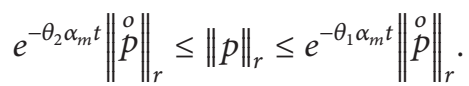

This inequality for $p=G_{F}(t) \stackrel{o}{p}$ yields

$$
e^{-\theta_{2} \alpha_{m} t}\left\|\begin{array}{l}
o \\
p
\end{array}\right\|_{r} \leq\left\|G_{F}(t) \stackrel{o}{p}\right\|_{r} \leq e^{-\theta_{1} \alpha_{m} t}\|\|_{p}^{o} \|_{r},
$$


where $\stackrel{o}{p} \in\left(C_{0}^{\infty}(\Lambda)\right)_{+} \subseteq D(F)_{+}$. If we take $0 \leq \stackrel{o}{p} \in \mathscr{X}_{r}$, then we can always mollify it by construction of approximations to the identity (mollifiers) $\omega_{\varepsilon}(\mathbf{v})=C_{\varepsilon} \omega(\mathbf{v} / \varepsilon)$ (as in [5, Example 2.1]), where $\omega$ is a $C_{0}^{\infty}(\Lambda)$ function defined by

$$
\omega(\mathbf{v})= \begin{cases}\exp \left(\frac{1}{|\mathbf{v}|^{2}-1}\right) & \text { for }|\mathbf{v}|<1 \\ 0 & \text { for }|\mathbf{v}| \geq 1\end{cases}
$$

and $C_{\varepsilon}$ are constants chosen so that $\int_{\Lambda} \omega_{\varepsilon}(\mathbf{v}) d x=1$.

Using the mollification of $\stackrel{o}{p}$ by taking the convolution

$$
\stackrel{o}{p_{\varepsilon}}:=\int_{\Lambda} \stackrel{o}{p}(\mathbf{v}-\mathbf{y}) \omega_{\varepsilon}(\mathbf{y}) d \mu_{y}=\int_{\Lambda} \stackrel{o}{p}(\mathbf{y}) \omega_{\varepsilon}(\mathbf{v}-\mathbf{y}) d \mu_{y}
$$

we obtain $\stackrel{o}{p_{\varepsilon}}$ in $\mathscr{X}_{r}$ (since $\stackrel{o}{p} \in \mathscr{X}_{r}$ ) and $\stackrel{o}{p}=\lim _{\varepsilon \rightarrow 0^{+}} \stackrel{o}{p_{\varepsilon}}$ in $\mathscr{X}_{r}$. Moreover, $\stackrel{o}{P_{\varepsilon}}$ are also nonnegative by (43) since $0 \leq \stackrel{o}{p}$, and the family $\left(\stackrel{o}{p_{\varepsilon}}\right)_{\varepsilon} \subseteq C_{0}^{\infty}(\Lambda)$. This shows that any nonnegative $\stackrel{o}{p}$ taken in $\mathscr{X}_{r}$ can be approximated by a sequence of nonnegative functions of $C_{0}^{\infty}(\Lambda)$. Inequality (41) is therefore valid for any nonnegative $\stackrel{o}{p} \in \mathscr{X}_{r}$. Using the fact that any arbitrary element $\stackrel{o}{g}$ of $\mathscr{X}_{r}$ (equipped with the pointwise order almost everywhere) can be written in the form $\stackrel{o}{g}=\stackrel{o}{g}_{+}-\stackrel{o}{g}_{-}$, where $\stackrel{o}{g}_{+}, \stackrel{o}{g}_{-} \in\left(\mathscr{X}_{r}\right)_{+}$, the positive element approach [18, 19] or [5, Theorem 2.64], allows us to extend the right inequality of (41) to all $\mathscr{X}_{r}$ so as to have

$$
\left\|G_{F}(t) p\right\|_{r} \leq e^{-\theta_{1} \alpha_{m} t}\|p\|_{r}
$$

Using the semigroup representation of the resolvent [5, Theorem 3.34], we obtain for $\lambda>0$

$$
\begin{aligned}
\|R(\lambda, F) p\|_{r} & \leq \int_{0}^{\infty} e^{-\lambda t}\left\|G_{F}(t) p\right\|_{r} d t \\
& \leq \int_{0}^{\infty} e^{-\lambda t} e^{-\theta_{1} \alpha_{m} t}\|p\|_{r} d t \\
& \leq \frac{1}{\lambda+\theta_{1} \alpha_{m}}\|p\|_{r} .
\end{aligned}
$$

By the right inequality of (35), we obtain that

$$
\|A R(\lambda, F) p\|_{r} \leq \frac{\theta_{2} \alpha_{m}}{\lambda+\theta_{1} \alpha_{m}}\|p\|_{r} \leq \frac{\theta_{2}}{\theta_{1}}\|p\|_{r} .
$$

This relation states that $D(A) \supseteq D(F)$ (the domain of $A$ is at least that of $F$ ). Because $F=\mathscr{D}-A$ and $A$ is bounded, we exploit the following relation for resolvent in $\mathscr{X}_{r}$ :

$$
\begin{gathered}
\lambda I-F=\lambda I-\mathscr{D}+A R(\lambda, F)(\lambda I-F), \\
I=(\lambda I-\mathscr{D}) R(\lambda, F)+A R(\lambda, F), \\
R(\lambda, \mathscr{D})=R(\lambda, F)+R(\lambda, \mathscr{D}) A R(\lambda, F), \\
R(\lambda, F)=R(\lambda, \mathscr{D})(I-A R(\lambda, F))
\end{gathered}
$$

for every $m \in \mathbb{R}_{+}$. This leads to $D(\mathscr{D}) \supseteq D(F)$, and therefore $D(F) \subseteq D(\mathscr{D}) \cap D(A)$.

On the other hand, if $p \in D(\mathscr{D}) \cap D(A)$ then $\|\mathscr{D} p\|_{r}<\infty$ and $\|A p\|_{r}<\infty$. Therefore

$$
\|\mathscr{D} p-A p\|_{r} \leq\|\mathscr{D} p\|_{r}+\|A p\|_{r}<\infty,
$$

meaning that $p \in D(F)$, and thus $D(\mathscr{D}) \cap D(A) \subseteq D(F)$, which ends the proof.

By the condition (35), the operator $A$ is the generator of a $C_{0}$-semigroup of contractions, let us say $\left(G_{A}(t)\right)_{t \geq 0}$. The following theorem holds.

Theorem 5. Assume that (35) is satisfied; then the operator $(F, D(F))$ is the generator of a substochastic semigroup $\left(G_{F}(t)\right)_{t \geq 0}$ given by

$$
\left[G_{F}(t) p\right](\mathbf{v})=\left[\lim _{v \rightarrow \infty}\left[G_{\mathscr{D}}\left(\frac{t}{v}\right) G_{A}\left(\frac{t}{v}\right)\right]^{v} p\right](\mathbf{v})
$$

for $p \in \mathscr{X}_{r}$ and $t>0$, where $\left(G_{\mathscr{D}}(t)\right)_{t \geq 0}$ is defined by (21).

Proof. First of all let us prove that $F$ is the generator of a substochastic semigroup $\left(G_{F}(t)\right)_{t \geq 0}$ in $\mathscr{X}_{r}$ given by

$$
G_{F}(t) p=\lim _{v \rightarrow \infty}\left[G_{\mathscr{D}}\left(\frac{t}{v}\right) G_{A}\left(\frac{t}{v}\right)\right]^{v} p
$$

for $p \in D(F)$.

We need to show that $\mathscr{D}$ and $A$ satisfy the conditions of Corollary 5.5 in the book by Pazy [20].

(a) We know by Theorem 2 and assumption (35) that $\mathscr{D}$ and $A$ are generators of positive semigroups of contractions; then

$$
\begin{gathered}
\left\|G_{\mathscr{D}}(t)\right\|_{r} \leq 1=1 e^{0 t}, \\
\left\|G_{A}(t)\right\|_{r} \leq 1=1 e^{0 t} \quad \forall t \geq 0 .
\end{gathered}
$$

Thus, $\mathscr{D}, A \in \mathscr{G}(1,0)$ and $G_{\mathscr{D}}(t) \geq 0, G_{A}(t) \geq 0$ for all $t \geq 0$.

(b) By Hille-Yosida Theorem [5, Theorem 3.5], $\mathscr{D}$ is closed and densely defined in $\mathscr{X}_{r}$, and because $\mathscr{X}_{r}=D(A) \supset D(\mathscr{D})$, we have $D(\mathscr{D}) \cap D(A)=D(\mathscr{D})$ is dense in $\mathscr{X}_{r}$.

(c) By the above condition (a), we can write

$$
\begin{aligned}
\left\|\left(G_{\mathscr{D}}(t) G_{A}(t)\right)^{v}\right\|_{r} & \leq\left\|G_{\mathscr{D}}(t)\right\|_{r}^{v}\left\|G_{A}(t)\right\|_{r}^{v} \\
& \leq 1 \\
& =1 e^{0 v t}, \quad v=1,2,3, \ldots
\end{aligned}
$$

(d) By the bounded perturbation theorem [5, Theorem 4.9], $\mathscr{D}-A$ is the generator of a positive semigroup of contractions since $\mathscr{D}$ generates a positive semigroups of contractions (Theorem 2), and $A$ is bounded (assumption (35)).

We know that $\lambda I-(\mathscr{D}-A): D(\mathscr{D}) \rightarrow \mathscr{X}_{r}$, and by HilleYosida Theorem, $\lambda I-(\mathscr{D}-A)$ must be invertible for some $\lambda>0$ and $(\lambda I-(\mathscr{D}-A))^{-1} \in \mathscr{L}\left(\mathscr{X}_{r}\right)$ (the space of bounded linear operators from $\mathscr{X}_{r}$ into $\left.\mathscr{X}_{r}\right)$. Then the range of $\lambda I-$ $(\mathscr{D}-A)=\mathscr{X}_{r}$. Thus $\lambda I-(\mathscr{D}-A)$ is densely defined in $\mathscr{X}_{r}$. 
All the conditions of Corollary 5.5 in [20] are satisfied by $\mathscr{D}$ and $A$; then $\bar{F}=\bar{D}-A=\mathscr{D}-A=F$ is the generator of a semigroup $\left(G_{F}(t)\right)_{t \geq 0}$ defined by

$$
\begin{aligned}
& {\left[G_{F}(t) p\right](\mathbf{v}) } \\
& \quad=\left[\lim _{v \rightarrow \infty}\left[G_{\mathscr{D}}\left(\frac{t}{v}\right) G_{A}\left(\frac{t}{v}\right)\right]^{v} p\right](\mathbf{v}), \quad p \in \mathscr{X}_{r},
\end{aligned}
$$

where we have used the fact that $\mathscr{D}-A$ is closed since it is the generator of a positive semigroup of contractions (HilleYosida Theorem).

Let us show that $\left(G_{F}(t)\right)_{t \geq 0}$ is substochastic. By $(50)$ and the above condition (a), we have $G_{F}(t) \geq 0$ for all $t \geq 0$ since $G_{F}(t) p$ is the limit of a sequence of elements of the positive cone of $\mathscr{X}_{r}$

$$
\left(\mathscr{X}_{r}\right)_{+}=\left\{g \in \mathscr{X}_{r} ; g \geq 0\right\} \text { which is closed. }
$$

Lastly, by (52) and (50), we have

$$
\begin{aligned}
\left\|G_{F}(t)\right\|_{r} & \leq \lim _{v \rightarrow \infty}\left\|G_{\mathscr{D}}(t)\right\|_{r}^{v}\left\|G_{A}(t)\right\|_{r}^{v} \\
& \leq 1
\end{aligned}
$$

for all $t \geq 0$.

Now we take the gain part of the fragmentation process defined by (9) with the coefficients satisfying the conservation law (3) and consider the perturbed transport equation

$$
\begin{gathered}
\frac{\partial}{\partial t} p=\mathscr{D} p-A p+B p, \\
\left.p\right|_{t=0}=\stackrel{o}{p} .
\end{gathered}
$$

Theorem 6. If the assumptions of Theorem 5 hold, then there is an extension $(\mathscr{K}, D(\mathscr{K}))$ of $(\mathscr{D}-A+B, D(\mathscr{D})$ that generates the smallest substochastic semigroup on $\mathscr{X}_{r}$, denoted by $\left(G_{\mathscr{K}}(t)\right)_{t \geq 0}$.

Proof. This theorem is a direct continuation of Theorem 5 by virtue of the substochastic semigroup theory in Kato's Theorem in $L_{1}$ (see [5, Corollary 5.17]). Because $D(B):=$ $D(A)$ (relation (9)), we have $D(B) \supset D(\mathscr{D}) \cap D(A)$. Thus, to apply Kato's Perturbation Theorem, we just need to show that, for all $p \in D(\mathscr{D}-A)_{+}=(D(\mathscr{D}))_{+}$,

$$
\int_{\Lambda}(\mathscr{D} p-A p+B p) d \mu \leq 0 .
$$

Since $p \in D(\mathscr{D})_{+}$and since $\|A p\|_{r}<\infty,\|B p\|_{r}<\infty$, then we can split (57) so as to get its left-hand side equal to

$$
\begin{aligned}
\int_{\Lambda} \mathscr{D} p d \mu & \\
+\int_{\mathbb{R}^{3}} \int_{0}^{\infty} m^{r}( & -a(x, m) p(t, x, m) \\
& \left.+\int_{m}^{\infty} b(x, s, m) a(x, s) p(x, s) d s\right) d m d x .
\end{aligned}
$$

The first term vanishes by the stochasticity (33) of the operator $\mathscr{D}$. For the other term, using the relations (11) and (12) yields

$$
\begin{aligned}
& \int_{\mathbb{R}^{3}} \int_{0}^{\infty} m^{r}(-a(x, m) p(t, x, m) \\
& \left.\quad+\int_{m}^{\infty} b(x, s, m) a(x, s) p(x, s) d s\right) d m d x \\
& \leq \int_{\mathbb{R}^{3}} \int_{0}^{\infty} m^{r}(-a(x, m) p(t, x, m)) d m d x \\
& \quad+\int_{\mathbb{R}^{3}} \int_{0}^{\infty} a(x, s) p(x, s) s^{r} d s d x \\
& =0,
\end{aligned}
$$

which proves the theorem.

\section{Concluding Remarks}

In this paper, we used the theory of strongly continuous semigroups of operators [20] to analyze the well posedness of an integrodifferential equation modelling convectionfragmentation processes. This work generalizes the preceding ones with the inclusion of the spatial transportation kernel which was not considered before. We proved that the combined fragmentation-transportation operator is the infinitesimal generator of a strongly continuous stochastic semigroup, thereby addressing the problem of existence of solutions for this model. However the full identification of the generator and characterization of its domain remain an open problem.

\section{References}

[1] M. Cai, B. F. Edwards, and H. Han, "Exact and asymptotic scaling solutions for fragmentation with mass loss," Physical Review A, vol. 43, no. 2, pp. 656-662, 1991.

[2] B. F. Edwards, M. Cai, and H. Han, "Rate equation and scaling for fragmentation with mass loss," Physical Review A, vol. 41, no. 10, pp. 5755-5757, 1990.

[3] J. Huang, B. F. Edwards, and A. D. Levine, "General solutions and scaling violation for fragmentation with mass loss," Journal of Physics A, vol. 24, no. 16, pp. 3967-3977, 1991.

[4] M. Kostoglou and A. J. Karabelas, "On the breakage problem with a homogeneous erosion type kernel," Journal of Physics A, vol. 34, no. 8, pp. 1725-1740, 2001.

[5] J. Banasiak and L. Arlotti, Perturbations of Positive Semigroups with Applications, Springer Monographs in Mathematics, Springer, London, UK, 2006.

[6] J. Carr, "Asymptotic behaviour of solutions to the coagulationfragmentation equations. I. The strong fragmentation case," Proceedings of the Royal Society of Edinburgh. Section A, vol. 121, no. 3-4, pp. 231-244, 1992.

[7] S. C. Oukouomi Noutchie, "Analysis of the effects of fragmentation-coagulation in planktology," Comptes Rendus Biologies, vol. 333, no. 11-12, pp. 789-792, 2010. 
[8] W. Wagner, "Explosion phenomena in stochastic coagulationfragmentation models," The Annals of Applied Probability, vol. 15, no. 3, pp. 2081-2112, 2005.

[9] E. D. McGrady and R. M. Ziff, “'Shattering' transition in fragmentation," Physical Review Letters, vol. 58, no. 9, pp. 892895, 1987.

[10] J. Banasiak, "Kinetic-type models with diffusion: conservative and nonconservative solutions," Transport Theory and Statistical Physics, vol. 36, no. 1-3, pp. 43-65, 2007.

[11] J. Banasiak, "Transport processes with coagulation and strong fragmentation," Discrete and Continuous Dynamical Systems. Series B, vol. 17, no. 2, pp. 445-472, 2012.

[12] A. Okubo and S. A. Levin, Diffusion and Ecological Problems: Modern Perspectives, vol. 14 of Interdisciplinary Applied Mathematics, Springer, New York, NY, USA, 2nd edition, 2001.

[13] P. Hartman, Ordinary Differential Equations, John Wiley \& Sons, New York, NY, USA, 1964.

[14] M. Tsuji, "On Lindelöf's theorem in the theory of differential equations," Japanese Journal of Mathematics, vol. 16, pp. 149-161, 1940.

[15] M. Volpato, "Sul problema di Cauchy per una equazione lineare alle derivate parziali del primo ordine," Rendiconti del Seminario Matematico della Università di Padova, vol. 28, pp. 153-187, 1958.

[16] S. Mizohata, The Theory of Partial Differential Equations, Cambridge University Press, New York, NY, USA, 1973.

[17] H. Tanabe, Equations of Evolutions, Pitman, London, UK, 1979.

[18] C. J. K. Batty and D. W. Robinson, "Positive one-parameter semigroups on ordered Banach spaces," Acta Applicandae Mathematicae, vol. 2, no. 3-4, pp. 221-296, 1984.

[19] K. Yosida, Functional Analysis, Springer, Berlin, Germany, 6th edition, 1980.

[20] A. Pazy, Semigroups of Linear Operators and Applications to Partial Differential Equations, vol. 44 of Applied Mathematical Sciences, Springer, New York, NY, USA, 1983. 


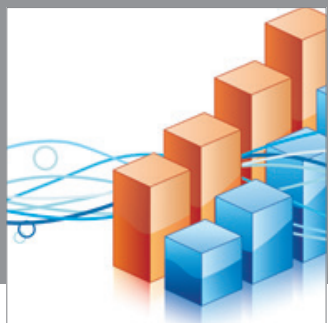

Advances in

Operations Research

mansans

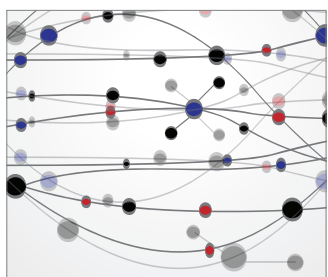

The Scientific World Journal
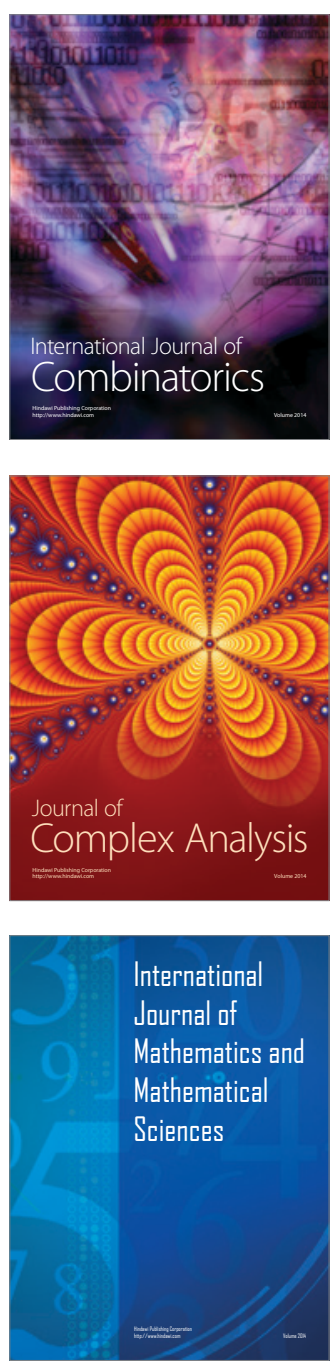
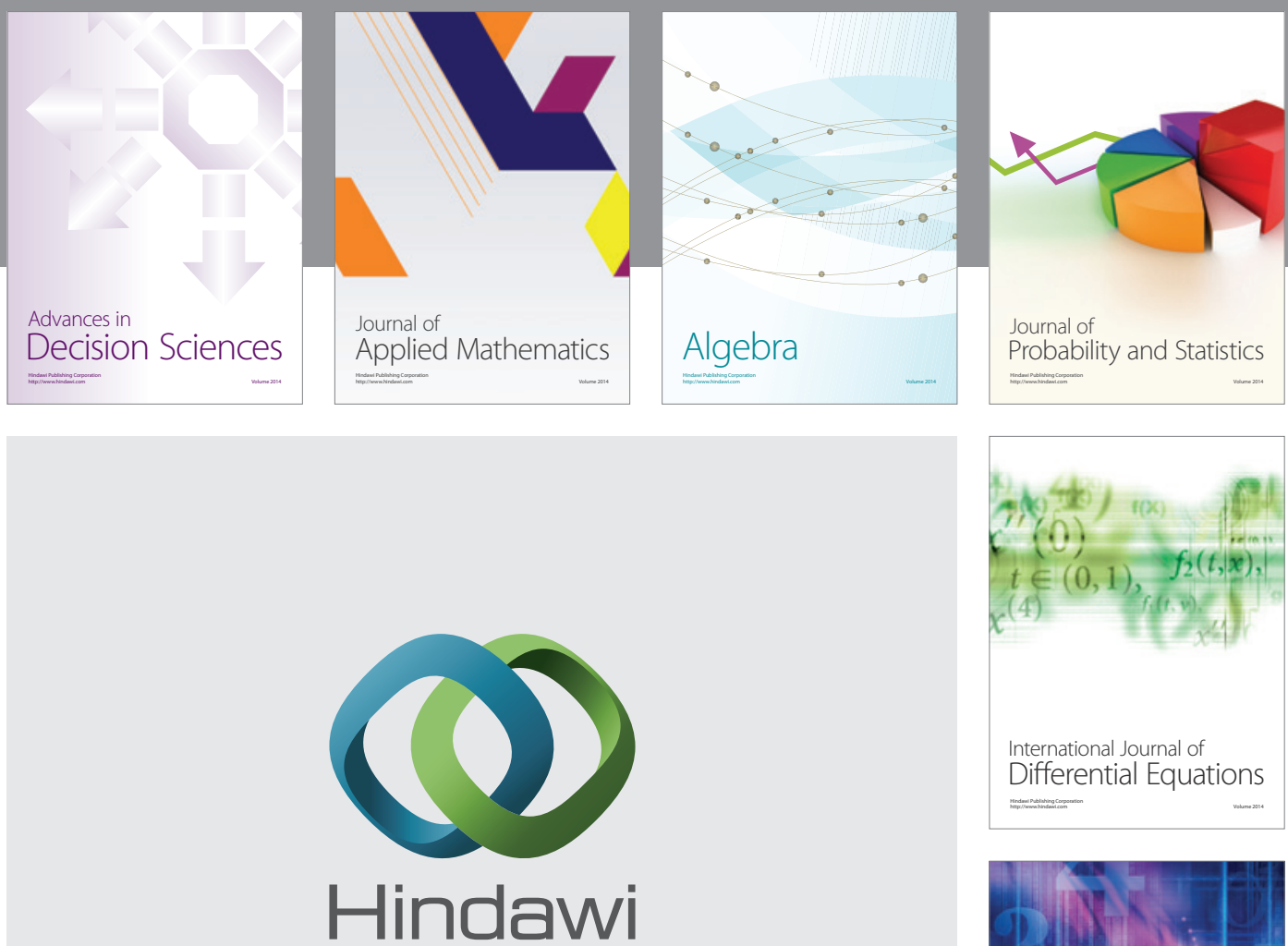

Submit your manuscripts at http://www.hindawi.com
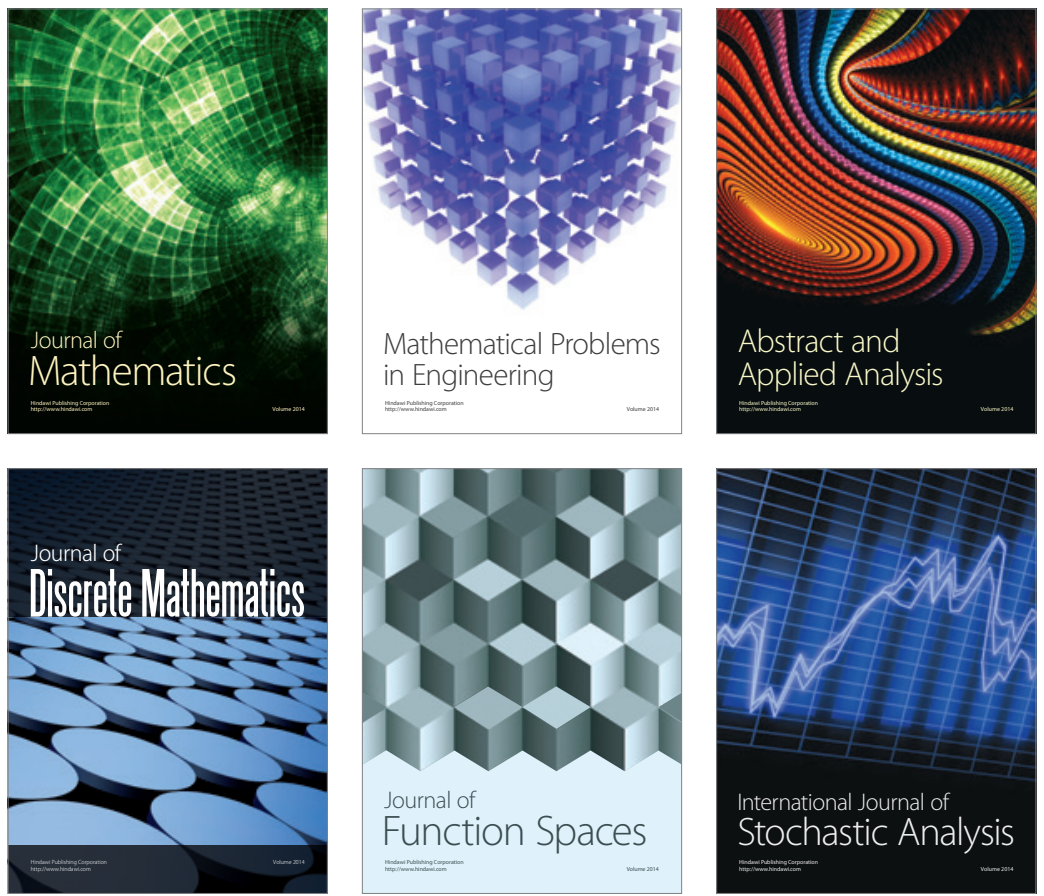

Journal of

Function Spaces

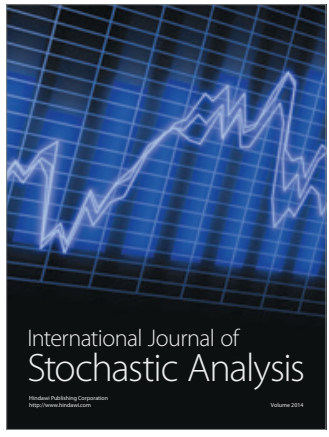

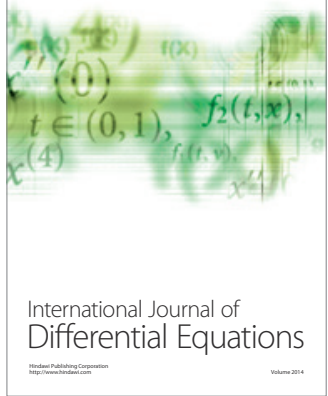
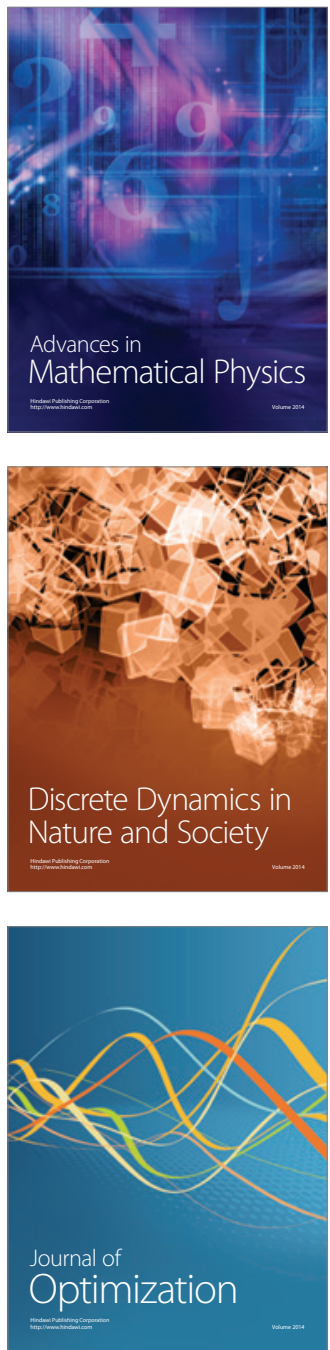